\title{
Generalized Radiation Boundary Conditions in Gyrotron Oscillator Modeling
}

\author{
S. Alberti ${ }^{1}$ - T. M. Tran $^{1}$ - S. Brunner ${ }^{1}$. \\ F. Braunmueller ${ }^{1} \cdot$ J. Genoud ${ }^{1} \cdot$ J.-Ph. Hogge ${ }^{1}$. \\ M. Q. Tran ${ }^{1}$
}

Received: 14 July 2015 / Accepted: 24 August 2015 /

Published online: 14 September 2015

(C) Springer Science+Business Media New York 2015

\begin{abstract}
A numerical procedure to implement a frequency-independent generalized non-reflecting radiation boundary conditions, GNRBC, based on the Laplace Transform, is described in details and tested successfully on a simple 2 frequency test problem. In the case of non-stationary regimes occurring in gyrotron oscillators, it is shown that the reflection at frequencies significantly separated from the carrier frequency can be effectively suppressed by this method. A detailed analysis shows that this numerical approach can be consistently used only for models in which there is no assumed separation of time scales between the RF field envelope timeevolution and the electron time of flight across the interaction region. The GNRBC has been implemented in a nonlinear time-dependent self-consistent monomode model, TWANGpic, in which there is no time scale separation since the RF field envelope is updated at each integration time step of the electron motion. The illustration of the effectiveness of the GNRBC is made with TWANGpic on a gyrotron for which extensive theoretical and experimental results have been performed.
\end{abstract}

Keywords Gyrotron oscillator · Radiation boundary conditions

\section{Introduction}

In the presence of non-stationary dynamics, a gyrotron oscillator is characterized by a frequency spectrum exhibiting the excitation of side-bands over a bandwidth $\Delta f / f$ of

S. Alberti

stefano.alberti@epfl.ch

1 Ecole Polytechnique Fédérale de Lausanne (EPFL), Centre de Recherches en Physique des Plasmas, CH-1015, Lausanne, Switzerland 
approximately $0.5-1 \%$ as shown in $[1,2]$ and references therein. The proper description of the wave-particle interaction in the commonly used gyro-averaged slow time scale models [3-5] requires to properly define the radiation boundary conditions at the extremities of the considered interaction space. Compared to the seminal paper by Ginzburg et al. [6], in which the non-reflecting frequency-independent boundary condition was derived and used in the case of a simplified interaction space with constant radius, in this paper, the interaction space is extended such to include the real gyrotron cavity as well as the uptaper and a simplified launcher following the cavity. This implies that the frequency-independent non-reflecting radiation boundary condition needs to be generalized (GNRBC) to the case in which it is defined at the launcher extremity with a radius significantly larger than the cavity constant radius section. This allows to consider cases in which the reference frequency (usually close to the cavity cutoff frequency) is significantly different than the cutoff frequency at the position of the GNRBC. An improved radiation boundary condition has also been considered by Wu et al. [7]. Considering this extended interaction space and a single transverse TE-mode, excited by the electron beam, non-stationary regimes can take place in the gyrotron cavity only $[1,2]$, but could possibly also occur in the launcher section at a frequency close to the cutoff frequency given by the launcher radius. In this case, the relative frequency separation between the frequencies excited in the cavity and the launcher can be as high as 8-10\%. A possible instability taking place in a simplified launcher geometry has been observed in some numerical codes and is called dynamic after-cavity interaction which underlying physical mechanism is described in [8] and references therein.

The paper is organized as follows: after the introduction, in Section 2 the underlying assumptions of the parabolic wave equation are recalled. In Section 3 the GNRBC is derived using a Laplace transform technique and it is shown that, compared to [6], an additional term appears in the form of a Fresnel integral. Section 4 describes in detail the numerical implementation of the GNRBC. For validating the numerical scheme, the results of the GNRBC are applied in Section 5 to a simple test case consisting of a constant radius waveguide excited with a source term containing two well separated frequencies. The GNRBC has been implemented in the nonlinear timedependent monomode model TWANGpic [9], and its effectiveness is illustrated and discussed in Section 6. Section 7 concludes the paper.

\section{Wave Envelope Equation}

In a cylindrical perfectly conducting waveguide with an adiabatically varying radius, the electric field associated to a single transverse eigenmode, $T E_{m, p}$, can be written as:

$$
\mathbf{E}(\mathbf{r}, t)=\operatorname{Re}\left[E(z, t) \mathbf{e}_{m, p}(r, \theta)\right],
$$

where $E(z, t)$ is the electric field amplitude and $\mathbf{e}_{m, p}(r, \theta)$ is a vector describing the transverse dependence of a given transverse mode at the axial position, $z$ [5]. 
The electric field amplitude, $E(z, t)$, satisfies the wave-equation:

$$
-\frac{1}{c^{2}} \frac{\partial^{2} E}{\partial t^{2}}+\frac{\partial^{2} E}{\partial z^{2}}-k_{\perp}^{2}(z) E=s,
$$

where the perpendicular wave vector $k_{\perp}(z)=v_{m, p} / R_{w}(z)$ is assumed to vary slowly along $z$ due to the slow variation of the cavity wall radius, $R_{w}(z)$. The quantity, $v_{m, p}$, is the $p^{t h}$ zero of the first derivative of the bessel function $J_{m}(x)$ and $s$ is a source term.

Assuming a reference frequency $\omega_{0}$ :

$$
E(z, t)=f(z, t) \exp \left(-i \omega_{0} t\right)
$$

the wave envelope equation (or parabolic wave equation) can be deduced from the full wave Eq. 2 as

$$
2 i \frac{\omega_{0}}{c^{2}} \frac{\partial f}{\partial t}+\frac{\partial^{2} f}{\partial z^{2}}+\left(\frac{\omega_{0}^{2}}{c^{2}}-k_{\perp}^{2}(z)\right) f=s \exp \left(i \omega_{0} t\right)
$$

where the term $\partial^{2} f / \partial t^{2}$ is neglected assuming $\left|\frac{1}{f} \frac{\partial f}{\partial t}\right| \ll \omega_{0}$. Finally, using the following time and space normalization

$$
\bar{t}=\omega_{0} t, \quad \bar{z}=k_{0} z=\frac{\omega_{0}}{c} z,
$$

the normalized envelope equation is given by

$$
2 i \frac{\partial f}{\partial \bar{t}}+\frac{\partial^{2} f}{\partial \bar{z}^{2}}+\left(1-k_{\perp}^{2} / k_{0}^{2}\right) f=s \exp (i \bar{t}) / k_{0}^{2} .
$$

Equation 6 needs to be completed with boundary conditions. For a gyrotron oscillator with the left boundary, $z=0$, in cut-off, the condition $f(0, \bar{t})=0$ is imposed. At the exit of the interaction region, at the right boundary, a radiation boundary condition is imposed and is described in the next section.

\section{Reflection Coefficient}

The analysis of the reflection at the waveguide boundary is conveniently performed in the wave frequency domain where the field envelope representation is simply the Laplace transform:

$$
F(\Omega, \bar{z})=\int_{0}^{\infty} d \bar{t} e^{i \Omega \bar{t}} f(\bar{t}, \bar{z}), \quad \operatorname{Im}(\Omega) \geq \gamma,
$$

with $\gamma$ being larger than any possible exponential growth rate of $f(\bar{t}, \bar{z})$.

The inverse Laplace transform can thus be defined as:

$$
f(\bar{t}, \bar{z})=\frac{1}{2 \pi} \int_{-\infty+i \gamma}^{\infty+i \gamma} d \Omega e^{-i \Omega \bar{t}} F(\Omega, \bar{z}) .
$$


The normalized frequency $\Omega$ defined in the Laplace transform (7) is related to the wave frequency $\omega$ by

$$
\omega=\omega_{0}(1+\Omega) .
$$

At a given axial position $z$, the field $F(\Omega)$ can be written as a sum of a forward and a backward wave:

$$
F(\Omega)=A_{+} \exp \left(i \kappa_{\|} \bar{z}\right)+A_{-} \exp \left(-i \kappa_{\|} \bar{z}\right),
$$

where the normalized parallel wave vector, $\kappa_{\|}$, is given by the local dispersion relation which can be deduced from Eq. 6 assuming $s=0$ :

$$
\kappa_{\|}^{2}(\bar{z})=2 \Omega+1-k_{\perp}^{2} / k_{0}^{2}=2 \Omega+\kappa_{\| 0}^{2},
$$

where we have introduced the axial wave vector $\kappa_{\| 0}$ at $\omega=\omega_{0}$ (or $\Omega=0$ ). Notice that this dispersion relation is the approximation of the full dispersion relation where $\Omega^{2}$ has been neglected, which is implied in the derivation of the envelope Eq. 6. From Eq. 10 and

$$
\frac{\partial}{\partial \bar{z}} F(\Omega)=i \kappa_{\|} A_{+} \exp \left(i \kappa_{\|} \bar{z}\right)-i \kappa_{\|} A_{-} \exp \left(-i \kappa_{\|} \bar{z}\right)
$$

the reflection coefficient can be obtained as

$$
R(\Omega)=\frac{A_{-}}{A_{+}}=\frac{i \kappa_{\|} F-\partial F / \partial \bar{z}}{i \kappa_{\|} F+\partial F / \partial \bar{z}} e^{2 i \kappa_{\|} \bar{z}} .
$$

A simplified non-reflecting boundary condition, SNRBC, for the envelope Eq. 6, assumes that the resulting stationary state has essentially a single frequency or a very narrow frequency spectrum peaked at the reference frequency, $\Omega=0$ :

$$
R(\Omega=0)=0 \Longrightarrow \frac{\partial F}{\partial \bar{z}}=i \kappa_{\| 0} F \Longrightarrow \frac{\partial f}{\partial \bar{z}}=i \kappa_{\| 0} f .
$$

This boundary condition leads however to non-zero reflection for $\Omega \neq 0$ :

$$
R(\Omega)=\frac{\kappa_{\|}-\kappa_{\| 0}}{\kappa_{\|}+\kappa_{\| 0}} e^{2 i \kappa_{\|} \bar{z}} .
$$

As shown in Fig. 1, a wave with $\Omega \simeq-0.06$ can have a reflection which is as large as $25 \%$.

In order to have $R(\Omega)=0$ for any $\Omega$, at the interaction region boundary, the non-reflecting boundary condition has to be imposed in the frequency domain as

$$
F(\Omega)=\frac{1}{i \kappa_{\|}} \frac{\partial F}{\partial \bar{z}}=\frac{1}{i \sqrt{2 \Omega+\kappa_{\| 0}^{2}}} \frac{\partial F}{\partial \bar{z}}(\Omega)=G(\Omega) \frac{\partial F}{\partial \bar{z}}(\Omega),
$$

having defined $G(\Omega)=1 / i \sqrt{2 \Omega+\kappa_{\| 0}^{2}}$.

Using the convolution theorem, the field in the time domain can be deduced as

$$
f(\bar{t})=\int_{0}^{\bar{t}} d \tau g(\tau) \frac{\partial f}{\partial \bar{z}}(\bar{t}-\tau)
$$




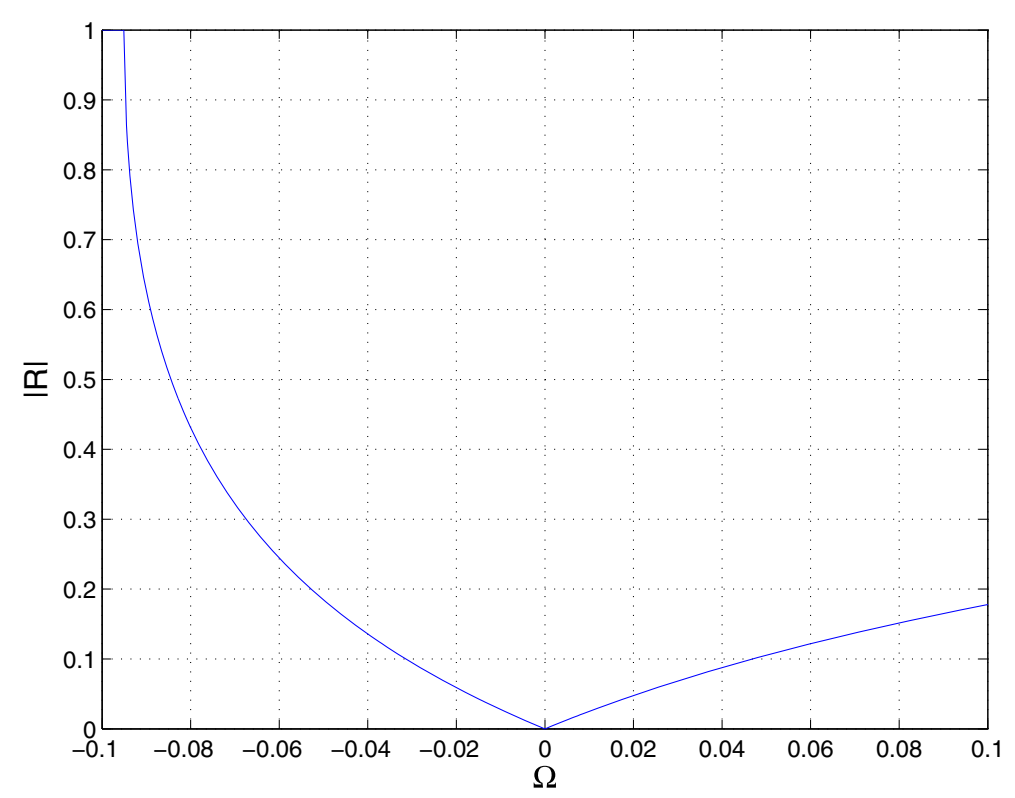

Fig. 1 The absolute value of the reflection coefficient as given by Eq. 15 for a SNRBC, Eq. 14 and $k_{\perp} / k_{0}=0.9$. The cut-off frequency is $\Omega_{c o}=-\kappa_{\| 0}^{2} / 2=-0.095$

where $g(\tau)$ is the inverse Laplace Transform of $G(\Omega)$ :

$$
g(\tau)=\frac{1}{2 \pi} \int_{-\infty+i \gamma}^{\infty+i \gamma} G(\Omega) e^{-i \Omega \tau} d \Omega=\frac{1}{2 \pi i} \int_{-\infty+i \gamma}^{\infty+i \gamma} \frac{e^{-i \Omega \tau}}{\sqrt{2 \Omega+\kappa_{\| 0}^{2}}} d \Omega, \quad \tau \geq 0,
$$

and, as shown in Appendix, can be obtained as:

$$
g(\tau)=\frac{1}{i} \sqrt{\frac{1}{2 \pi i \tau}} \exp \left(i \frac{\kappa_{\| 0}^{2}}{2} \tau\right), \quad \tau \geq 0 .
$$

One can readily check that

$$
\int_{0}^{\infty} d \tau g(\tau) e^{i \Omega \tau}=\frac{1}{i \sqrt{2 \Omega+\kappa_{\| 0}^{2}}}=G(\Omega) .
$$

The GNRBC for any frequency $\Omega$ can thus be expressed in the time domain as

$$
i \kappa_{\| 0} f(\bar{t})=\frac{\kappa_{\| 0}}{\sqrt{2 \pi i}} \int_{0}^{\bar{t}} \frac{d \tau}{\sqrt{\tau}} \exp \left(i \frac{\kappa_{\| 0}^{2}}{2} \tau\right) \frac{\partial f}{\partial \bar{z}}(\bar{t}-\tau) .
$$

If $\partial f / \partial \bar{z}$ is time-independent or weakly time-dependent (see [11]), one obtains

$$
\lim _{\bar{t} \rightarrow \infty} f(\bar{t})=\frac{\partial f}{\partial \bar{z}} \int_{0}^{\infty} d \tau g(\tau)=G(0) \frac{\partial f}{\partial \bar{z}}=\frac{1}{i \kappa_{\| 0}} \frac{\partial f}{\partial \bar{z}} .
$$

which is the single frequency approximation, Eq. 14. 
The same method has been proposed in [6] but the article considers the special case where the reference frequency $\omega_{0}$ is chosen as the cut-off frequency at the boundary, which implies that $\kappa_{\| 0}=0$.

\section{Numerical Implementation}

From here on, for notational convenience, the bar symbol is omitted on the normalized $t$ and $z$. Let us consider the discrete times $t_{j}=j \Delta t, j=0,1, \ldots$ and denote the field and its axial derivative defined at $t_{j}$ as $f_{j}$ and $f_{j}^{\prime}$, respectively. The GNRBC given by Eq. 21 can thus be approximated at the time $t_{n}$, up to second order in $\Delta t$ as:

$$
\begin{aligned}
i \kappa_{\| 0} f_{n}(t) & =\frac{\kappa_{\| 0}}{\sqrt{2 \pi i}} \sum_{j=1}^{n} \int_{(j-1) \Delta t}^{j \Delta t} d \tau \frac{e^{i \frac{\kappa_{\| 0}^{2}}{2} \tau}}{\sqrt{\tau}} f^{\prime}\left(t_{n}-\tau\right) \\
& \simeq \sum_{j=1}^{n} \frac{f_{n-j+1}^{\prime}+f_{n-j}^{\prime}}{2}\left(A_{j}-A_{j-1}\right),
\end{aligned}
$$

where $f^{\prime}(t)$ is approximated as piece-wise constant:

$$
f^{\prime}(t) \simeq \frac{f^{\prime}(j \Delta t)+f^{\prime}((j-1) \Delta t)}{2}=\frac{f_{j}^{\prime}+f_{j-1}^{\prime}}{2} \text {, for } t \in[(j-1) \Delta t, j \Delta t] .
$$

The quantity $A_{j}$ in Eq. 23 is given by:

$$
A_{j}=\frac{\kappa_{\| 0}}{\sqrt{2 \pi i}} \int_{0}^{j \Delta t} d \tau \frac{e^{i \frac{\kappa_{\| 0}^{2}}{2} \tau}}{\sqrt{\tau}}=\sqrt{\frac{2}{i}}[C(\beta \sqrt{j})+i S(\beta \sqrt{j})],
$$

where $C(z)$ and $S(z)$ are the Fresnel Integrals [13]:

$$
C(z)=\int_{0}^{z} \cos \left(\frac{\pi}{2} t^{2}\right) d t, \quad S(z)=\int_{0}^{z} \sin \left(\frac{\pi}{2} t^{2}\right) d t
$$

and

$$
\beta=\kappa \| 0 \sqrt{\Delta t / \pi} .
$$

Finally, the GNRBC can be written as a convolution sum

$$
\begin{aligned}
i \kappa_{\| 0} f_{n} & =\sum_{j=0}^{n} \chi_{j} f_{n-j}^{\prime} \\
\chi_{0} & =\frac{1}{2}\left(A_{1}-A_{0}\right), \\
\chi_{j} & =\frac{1}{2}\left(A_{j+1}-A_{j-1}\right), \quad j=1,2, \ldots, n-1, \\
\chi_{n} & =\frac{1}{2}\left(A_{n}-A_{n-1}\right) .
\end{aligned}
$$


Notice that

$$
\lim _{n \rightarrow \infty} \sum_{j=0}^{n} \chi_{j}=\lim _{n \rightarrow \infty} \frac{A_{n+1}+A_{n}}{2}=\sqrt{\frac{2}{i}} \lim _{x \rightarrow \infty}[C(x)+i S(x)]=1,
$$

clearly recovering Eq. 22.

\section{A Simple Test Case}

To test the efficiency of the GNRBC described above, a constant radius simple cavity (constant $k_{\perp}$ ) of length $L$ and is driven by the LHS boundary condition at the input, $z=0$ :

$$
f(z=0, t)=\left[1+\exp \left(-i \Omega_{1} t\right)\right] \min \left(1, t / t_{r}\right),
$$

which defines a two-frequency driving term, with the first frequency at $\Omega=0$ and the second at $\Omega_{1}$. The GNRBC is imposed at $z=L$.

Using a finite element approach, the weakform of Eq. 6 and the following B-spline [12] expansion for $f$ and its derivative $f^{\prime}=\partial f / \partial z$ :

$$
\begin{aligned}
f(z, t) & =\sum_{\nu=1}^{N} c_{\nu}(t) \Lambda_{\nu}(z), \\
f^{\prime}(z, t) & =\sum_{\nu=1}^{N} c_{\nu}(t) \Lambda_{\nu}^{\prime}(z),
\end{aligned}
$$

where $N=N_{z}+p, N_{z}$ is the number of intervals along $z$ and $p$ the order of the B-splines, the following system of ODE's can be derived for the field coefficients c(t):

$$
\begin{gathered}
\mathbf{M} \frac{d \mathbf{c}}{d t}+\mathbf{N} \cdot \mathbf{c}=0, \\
M_{v v^{\prime}}=2 i \int_{0}^{L} d z \Lambda_{v} \Lambda_{v^{\prime}}, \quad N_{v v^{\prime}}=\int_{0}^{L} d z\left(-\Lambda_{v}^{\prime} \Lambda_{v^{\prime}}^{\prime}+\kappa_{\| 0}^{2} \Lambda_{v} \Lambda_{v^{\prime}}\right) .
\end{gathered}
$$

Using a second order time centered implicit discretization, the following linear system of equations for the field $\mathbf{c}^{n}$ at time $t_{n}=n \Delta t$ is obtained:

$$
\begin{aligned}
& \mathbf{M}\left(\frac{\mathbf{c}^{n}-\mathbf{c}^{n-1}}{\Delta t}\right)+\mathbf{N}\left(\frac{\mathbf{c}^{n}+\mathbf{c}^{n-1}}{2}\right)=0, \\
\Longrightarrow & \left(\mathbf{M}+\frac{\Delta t}{2} \mathbf{N}\right) \mathbf{c}^{n}=\left(\mathbf{M}-\frac{\Delta t}{2} \mathbf{N}\right) \mathbf{c}^{n-1} .
\end{aligned}
$$

The zero initial condition can be imposed simply by starting with $\mathbf{c}^{0}=0$ while the essential boundary conditions at $z=0$, Eq. 30 and at $z=L$, Eq. 28 are implemented by replacing respectively the first and last equations of the linear system above with:

$$
\begin{gathered}
c_{1}^{n}=\left[1+\exp \left(-i \Omega_{1} t_{n}\right)\right] \min \left(1, t_{n} / t_{r}\right) \\
i \kappa_{\| 0} c_{N}^{n}-\chi_{0} \sum_{\nu=N-p}^{N} \Lambda_{\nu}^{\prime}(L) c_{\nu}^{n}=\sum_{j=1}^{n} \chi_{j} f^{\prime}\left(L, t_{n}-j \Delta t\right) .
\end{gathered}
$$


Notice that with this non reflecting boundary condition, the time marching algorithm involves all the previously computed values of $f^{\prime}$ at $z=L$.

Figure 2 shows the results obtained with the simple boundary conditions (14). From left to right and top to bottom are shown the early time evolution of $f$ at $z=0$ and $z=L$, the real and imaginary parts as well as the modulus and phase of the complex field versus $z$ at the stationary state. The last two figures are obtained by performing a discrete Fourier transform of the field at $z=L$ on a suitable chosen
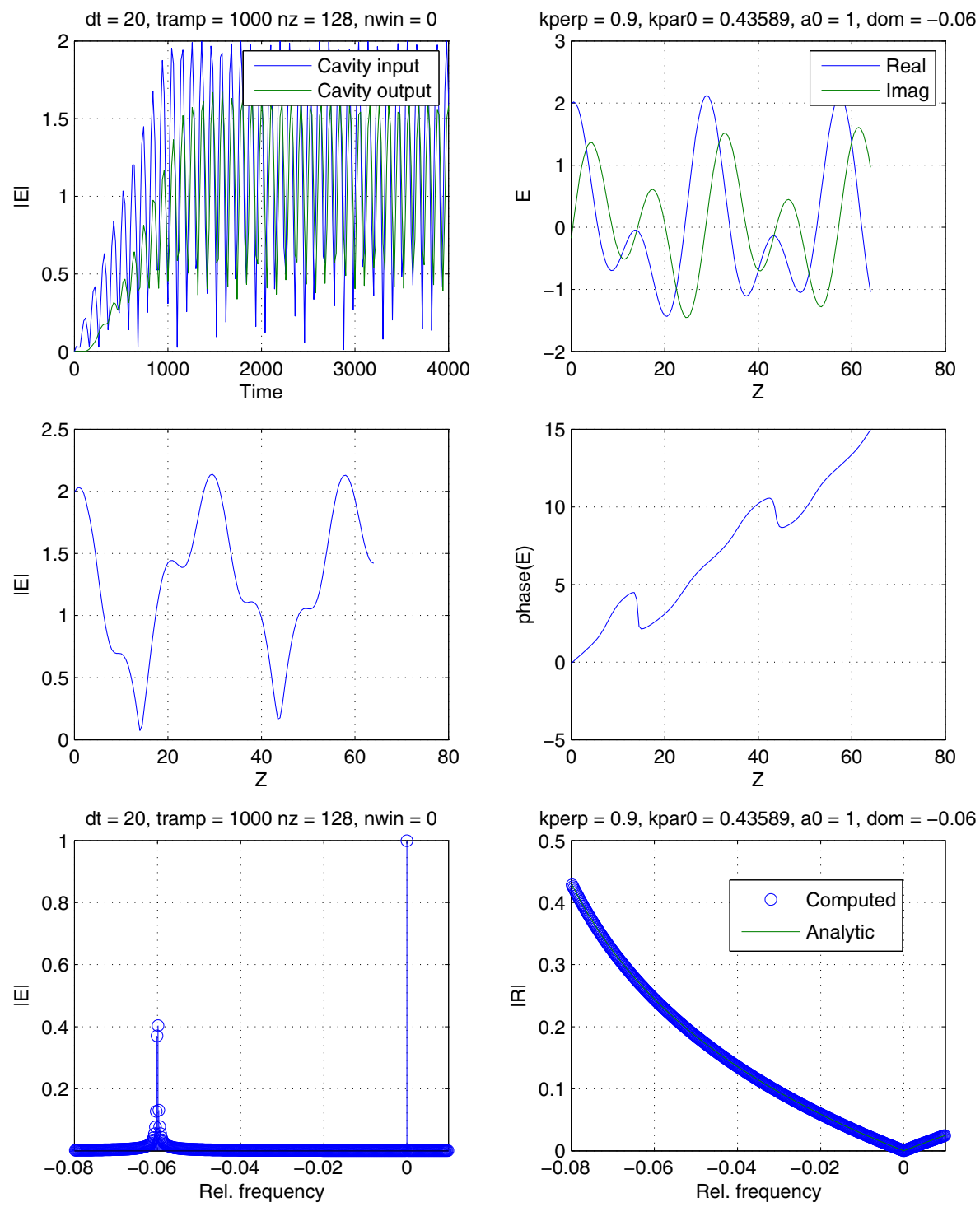

Fig. 2 Results from the simple test problem for a SNRBC, Eq. 15 and $k_{\perp} / k_{0}=0.9$ 
time window and the definition of the frequency-dependent reflection coefficient in Eq. 13. As expected, the computed reflection here agrees very well with Eq. 15 and shows a high reflection $(\approx 25 \%)$ at the drive frequency $\Omega_{1}=-0.06$.

Using the numerical implementation of the GNRBC given in Eq. 34, one can reduce this reflection to less than $1 \%$ as shown in Fig. 3, resulting in a field axial profile closer to a cosine function. Figure 4 shows that to achieve very low
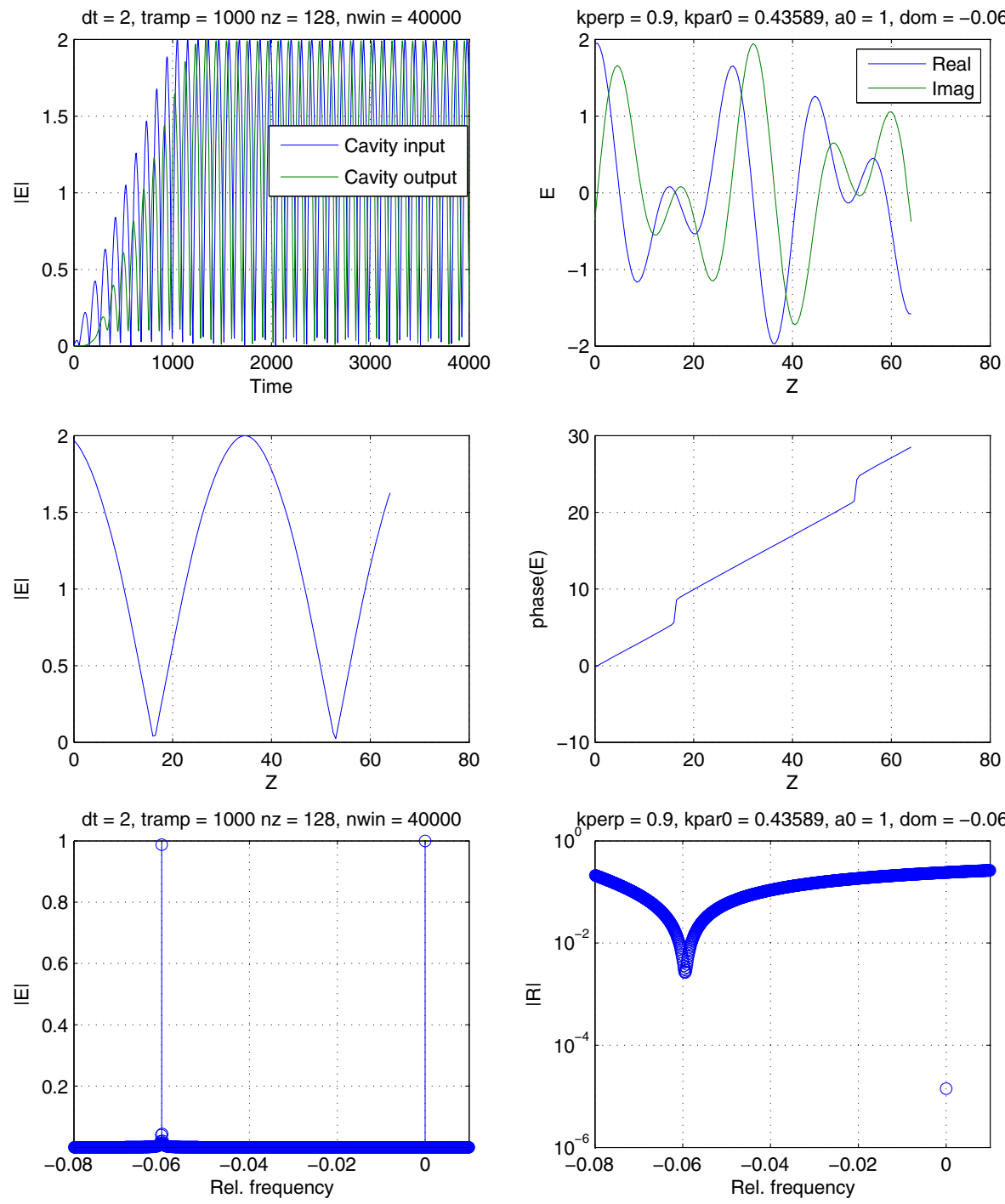

Fig. 3 Results from the simple test problem using the GNRBC, Eq. $28, k_{\perp} / k_{0}=0.9,\left(\omega_{1}-\omega_{0}\right) / \omega_{0}=$ -0.06 and $\omega_{0} \Delta t=2$. Note that compared to Fig. 2, for highlighting the effect of GNRBC, the amplitude of the reflection coefficient is in logarithmic scale and the isolated point at $\Omega=0$ corresponds to the calculated reflection coefficient at this frequency 


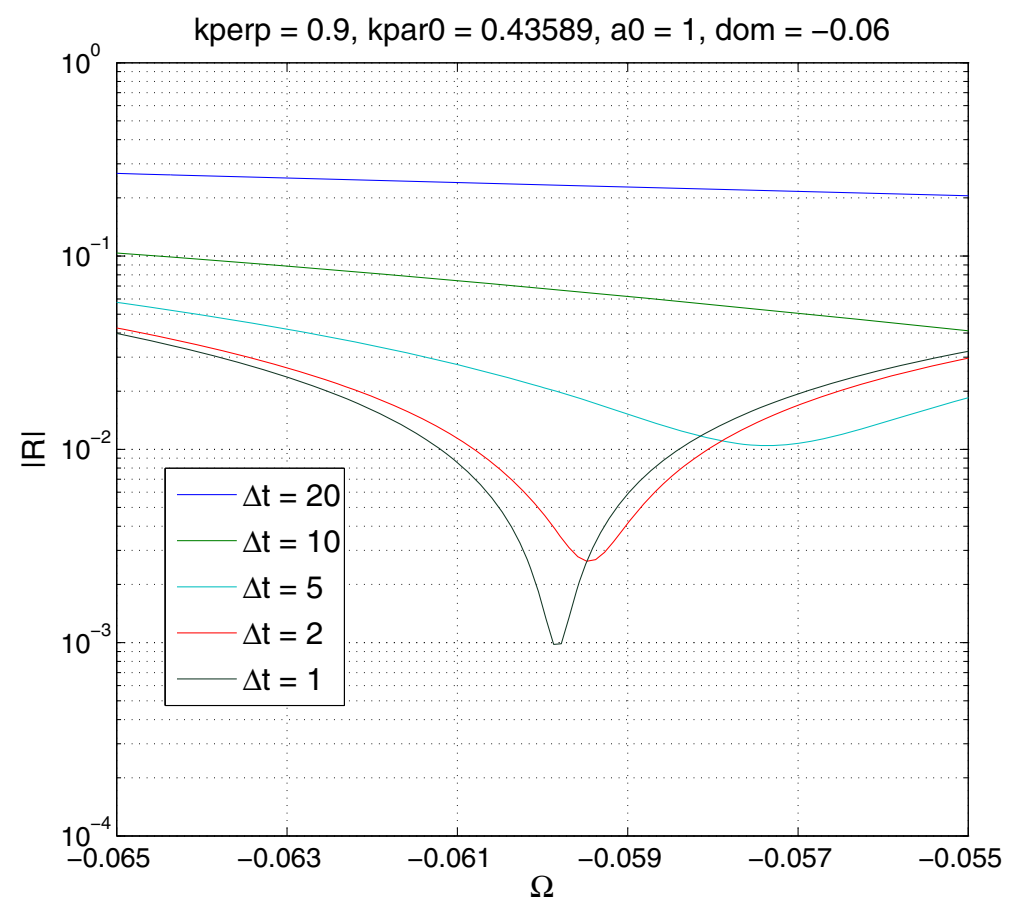

Fig. 4 The reflection coefficient $R(\Omega)$ in the vicinity of $\Omega_{1}=\left(\omega_{1}-\omega_{0}\right) / \omega_{0}=-0.06$ for different time steps $\omega_{0} \Delta t$ and $k_{\perp} / k_{0}=0.9$

reflection, the time step should be small enough to resolve accurately the drive frequency $\Omega_{1}$. This is confirmed in Fig. 5 where the convergence of the reflection at the drive frequency $\Omega_{1}$ with respect to $\left|\Omega_{1}\right| \Delta t$ is examined for $\Omega_{1}=-0.06$ and $\Omega_{1}=-0.03$. Finally, Fig. 6 shows that this reduction of the reflection (at the drive frequency $\Omega_{1}=-0.06$ ) is achieved through a steady reduction of the amplitude of the backward wave $A_{-}$while the forward wave $A_{+}$remains almost unchanged.

\section{Illustration of GNRBC in the TWANGpic Simulation Codes}

To illustrate the effect of the GNRBC, the example of the $140 \mathrm{GHz}$ gyrotron developed for the W7-X stellarator is considered [10, 14, 15] for which realistic experimental parameters are used. The GNRBC is implemented in the TWANGpic [9] code which is based on 1D Particle-in-Cell (PIC) approach. The essential feature of TWANGpic is the fact that there is no time-scale separation between the evolution of the rf-wave envelope, and the particles in the sense that the rf-field envelope is updated at each integration time step of the electron motion: the wave-particle interaction is described by a one-dimensional Particle-in-Cell (PIC) approach. This feature not only allows to study non-stationary dynamics in gyrotron oscillators, but 


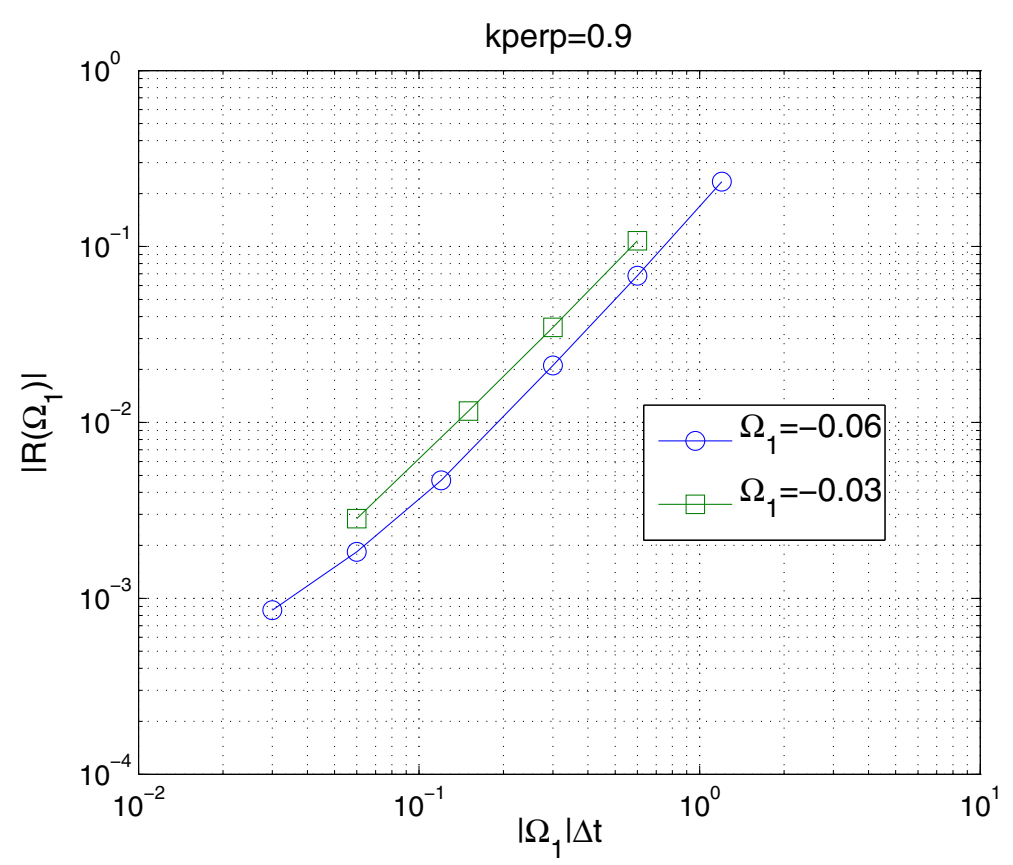

Fig. 5 Convergence of $R\left(\Omega_{1}\right)$ with respect to $\left|\omega_{1}-\omega_{0}\right| \Delta t$ for $k_{\perp} / k_{0}=0.9$, and the two drive frequencies $\Omega_{1}=-0.06$ and $\Omega_{1}=-0.03$

also is consistent with the time step required for exploiting the GNRBC which is the subject of this paper.

The interaction space wall-radius profile as well as the magnetic field profile are shown in Fig. 7 (top). Note that for the purpose of this study on GNRBC, a simplified launcher geometry $(80 \mathrm{~mm}<z<140 \mathrm{~mm}$ ) has been considered which does not include any helical perturbations as it is the case for the real launcher described in [14] and references therein. The main system parameters are the cavity mode is the $T E_{28,8}$, magnetic field maximum $B_{0}=5.6152 T$ at $z=25 \mathrm{~mm}$, beam energy $E_{b}=$ $81.8 \mathrm{keV}$, beam current $I_{b}=43.2 \mathrm{~A}$, pitch angle $\alpha=1.18$ with no alpha-spread, and guiding radius $r_{g 0}=10.17 \mathrm{~mm}$.

The self-consistent rf-field amplitude and phase profiles across the interaction space, calculated with TWANGpic, are shown in Fig. 7. In both figures (middle for the amplitude and bottom for the phase), the comparison of the results obtained with the SNRBC (red curve) and GNRBC (dashed blue curve) is made. In the interaction space defined by the cavity, $z \leq 0.05 \mathrm{~m}$, the results for the two boundary conditions are identical. However, after the cavity, in the simplified launcher section $(0.08 m \leq z \leq 0.14 m)$, one clearly observes, both on the amplitude and phase a significant difference between the two boundary conditions, with in particular a constant amplitude and a linear phase for the GNRBC case.

Considering the time trace of the real part of the rf electric field at the interaction space exit $(z=0.14 \mathrm{~m})$, one observes in Fig. 8 that for the SNRBC (green curve), 


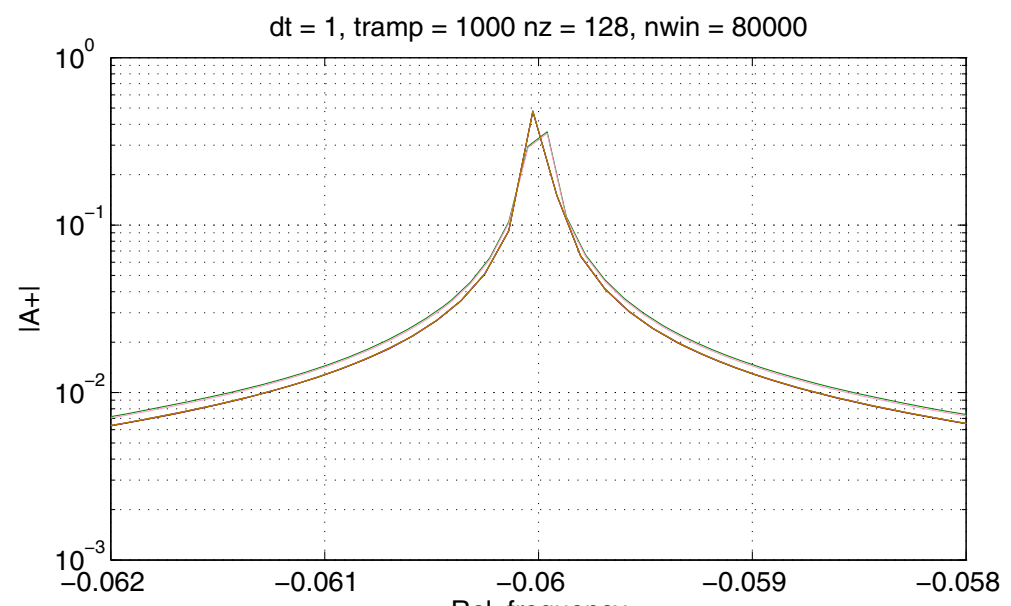

Rel. frequency

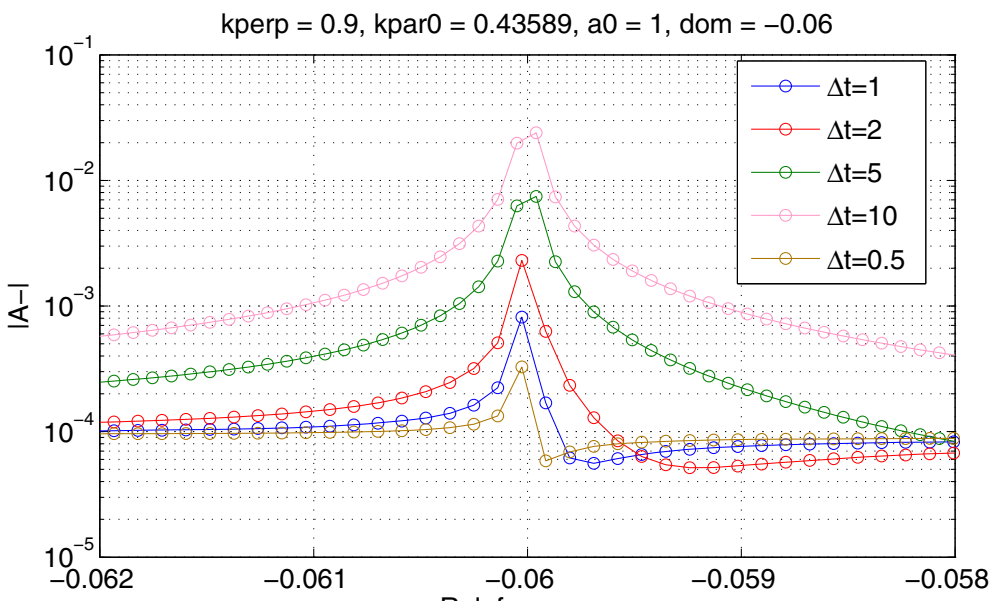

Rel. frequency

Fig. 6 Changes of forward and backward wave amplitudes as $\omega_{0} \Delta t$ decreases for $k_{\perp} / k_{0}=0.9$ and $\Omega_{1}=-0.06$

on top of the main frequency, excited in the cavity, a second frequency is excited at the launcher exit. This second frequency does not appear when the GNRBC is used, therefore, demonstrating that this spurious frequency is a numerical artifact associated to the boundary condition used in the model.

Considering the rf-field at the radiation boundary $(z=0.14 \mathrm{~m})$ shown in Fig. 8, the associated frequency spectrum is shown in Fig. 9 (top) which clearly shows that for the case using the SNRBC the second frequency excited at the exit of the launcher section is approximately $8 \mathrm{GHz}$ lower than the main frequency at $140 \mathrm{GHz}$. With such an important frequency separation, when the SNRBC is used, an important reflection $(|R| \approx 25 \%)$ is generated at the radiation boundary condition for the spurious frequency at $\Omega=-0.06$ (see Fig. 1). 

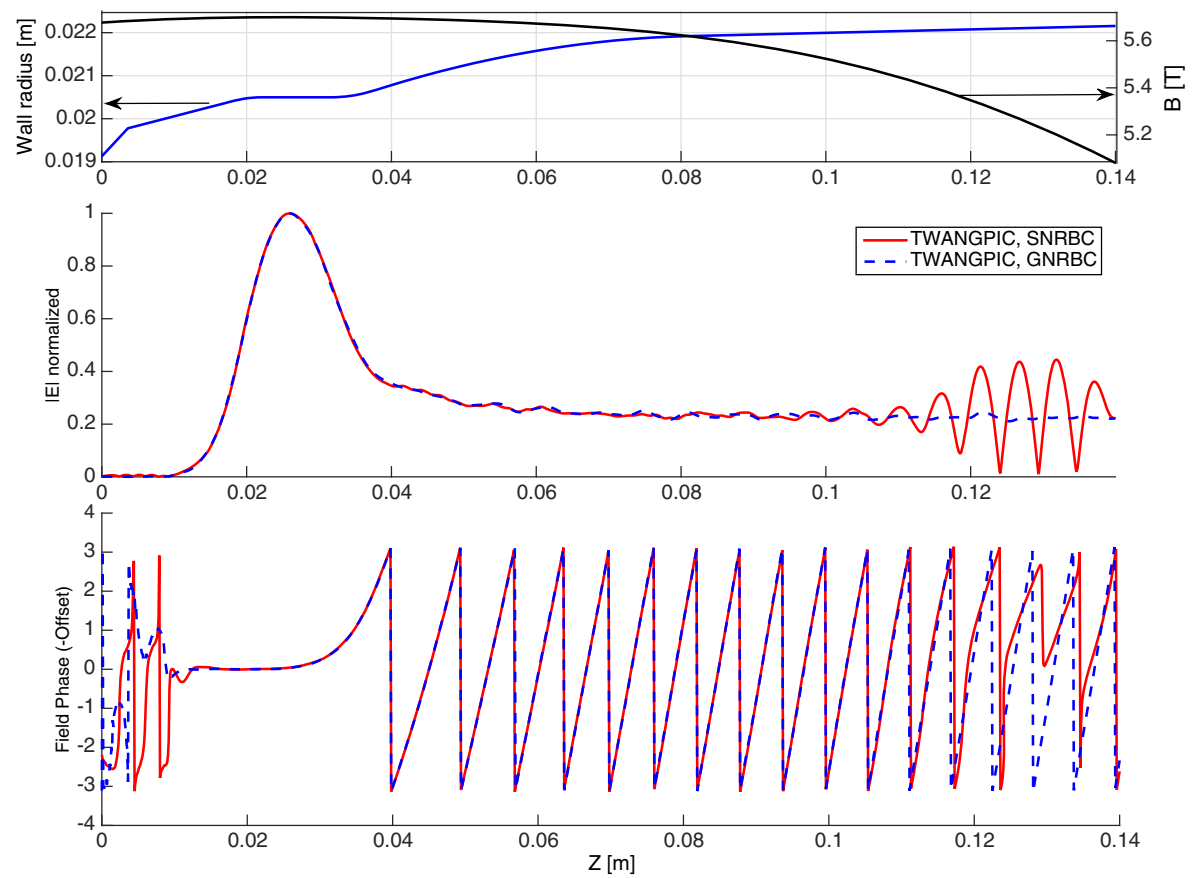

Fig. $7140 \mathrm{GHz}$ gyrotron for W7-X. Top: Wall radius and magnetic field profiles considered for the simulations. The cylindrical wall radius includes the cavity, the uptaper and a simplified geometry of the launcher without helical wall perturbations. Middle and bottom Self-consistent amplitude (middle) and phase (bottom) profiles calculated with TWANGpic with GNRBC (dashed blue) and without (red)

The decomposition of the rf-field in backward and forward waves is shown in Fig. 9 (middle) for the SNRBC and Fig. 9 (bottom) for the GNRBC and for the frequency domain in which the spurious frequency is excited when using the SNRBC.

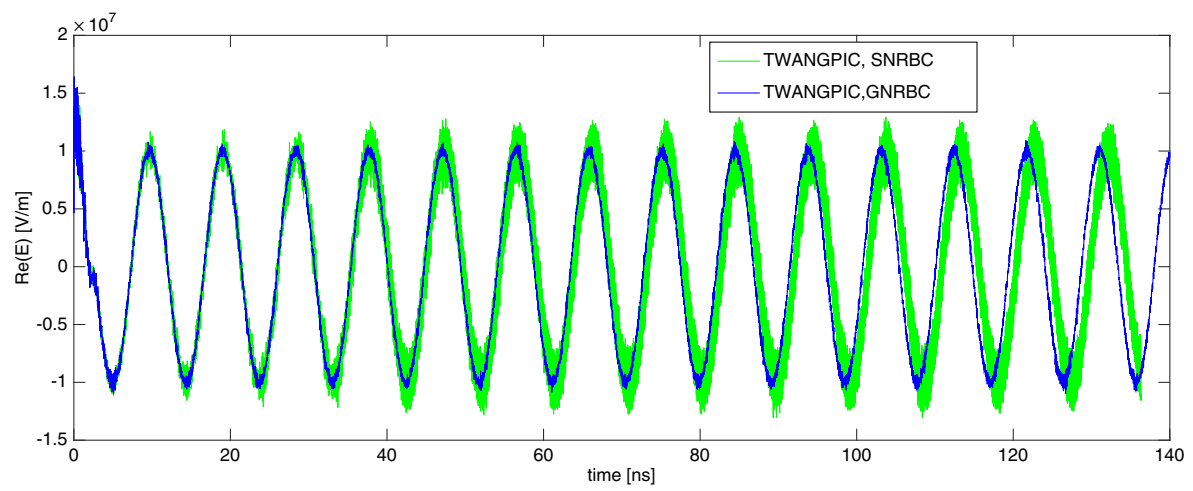

Fig. $8140 \mathrm{GHz}$ gyrotron for W7-X. Real part of the rf-electric field computed with TWANGpic at the exit of the interaction region, $z=0.14 \mathrm{~m}$. In blue with the GNRBC and in green without 

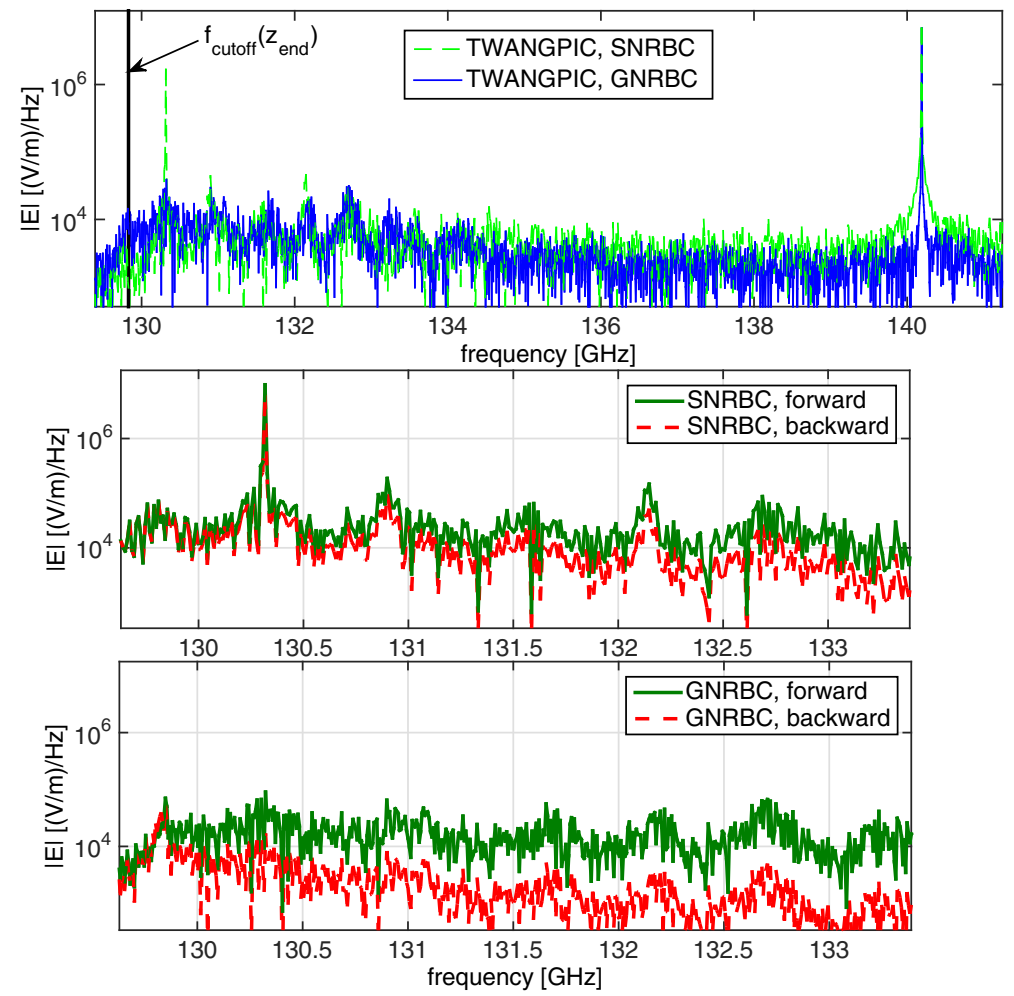

Fig. $9140 \mathrm{GHz}$ gyrotron for W7-X. Top: rf-field frequency spectrum calculated with TWANGpic at the exit of the interaction space with (blue) and without (dashed green) GNRBC. The black vertical line indicates the cut-off frequency calculated at the exit of the interaction space $z=140 \mathrm{~mm}$. Middle: forward and backward waves rf-field spectrum in the frequency range where the dynamic-ACI instability appears when using SNBRC Bottom: same as the middle figure, but with GNRBC. No dynamic-ACI is observed with GNRBC

The effectiveness of the GNRBC is clearly visible since no backward wave component exists when GNRBC is considered. The excitation of this spurious frequency in the launcher region is called dynamic After Cavity Instability (dynamic-ACI). Its detailed analysis is outside the scope of this paper and is described in [8].This example stresses the fact that, even though dynamic-ACI is physically plausible, in many cases, it is a numerical artifact associated to the model describing the waveparticle interaction and in particular the implemented radiation boundary condition. As shown, by using the GNRBC, the spurious frequency excitation disappears.

\section{Conclusion}

A numerical scheme defining a generalized non-reflecting frequency independent radiation boundary condition, GNRBC, has been successfully implemented in the 
monomode self-consistent TWANGpic code. The model derivation, its numerical implementation as well as a detailed numerical study of its properties on a simple test case has been presented. The effectiveness of GNRBC has been demonstrated on simulations performed on a gyrotron cavity extensively studied both in theory and experiments.

Acknowledgments This work is partially supported by EFDA under Grant WP13-DAS-HCD-EC and by Fusion for Energy under Grants F4E-GRT-432 and -553 within the European Gyrotron Consortium (EGYC). The views and opinions expressed herein do not necessarily reflect those of the European Commission. EGYC is a collaboration of CRPP, Switzerland; KIT, Germany; HELLAS, Greece; IFP-CNR, Italy. The authors would like to thank Dr. K. Avramides from KIT (Karlsruhe Institute for Technology) for precious scientific discussions.

\section{Appendix: Inverse Laplace Transform of $1 / i \kappa_{\|}(\Omega)$}

Using the dispersion relation (11), the inverse Laplace transform can be computed as follow

$$
g(\tau)=\frac{1}{2 \pi i} \int_{-\infty+i \gamma}^{\infty+i \gamma} \frac{e^{-i \Omega \tau}}{\sqrt{2 \Omega+\kappa_{\| 0}^{2}}} d \Omega=\frac{e^{i \frac{\kappa_{\| 0}^{2}}{2} \tau}}{4 \pi i} \int_{-\infty+i \gamma}^{\infty+i \gamma} \frac{e^{-i \Omega \tau / 2}}{\sqrt{\Omega}} d \Omega
$$

Splitting the integral path between its $\operatorname{Re}(\Omega)<0$ and $\operatorname{Re}(\Omega)>0$ parts, and noting that

$$
\sqrt{\Omega}=\sqrt{-(-\Omega)}=i \sqrt{-\Omega}, \quad \text { for } \operatorname{Re}(\Omega)<0,
$$

the integral becomes, in the limit $\gamma \rightarrow 0$

$$
\int_{-i \gamma}^{\infty-i \gamma} d \Omega \frac{e^{i \tau \Omega / 2}}{i \sqrt{\Omega}}+\int_{i \gamma}^{\infty+i \gamma} d \Omega \frac{e^{-i \tau \Omega / 2}}{\sqrt{\Omega}}=\sqrt{2 \pi}\left[-i \sqrt{\frac{1}{-i \tau}}+\sqrt{\frac{1}{i \tau}}\right],
$$

where we have used the following result

$$
\int_{0}^{\infty} d u e^{-z u^{2}}=\frac{1}{2} \sqrt{\frac{\pi}{z}}, \quad \operatorname{Re}(z) \geq 0 .
$$

For $\tau<0$, the sum inside the bracket can be calculated as

$$
-i \sqrt{\frac{1}{i|\tau|}}+\sqrt{\frac{1}{-i|\tau|}}=\frac{1}{\sqrt{|\tau|}}\left(\frac{e^{-i \pi / 2}}{e^{i \pi / 4}}+\frac{1}{e^{-i \pi / 4}}\right)=0
$$

while for $\tau>0$

$$
-i \sqrt{\frac{1}{-i|\tau|}}+\sqrt{\frac{1}{i|\tau|}}=\frac{1}{\sqrt{|\tau|}}\left(\frac{e^{-i \pi / 2}}{e^{-i \pi / 4}}+\frac{1}{e^{i \pi / 4}}\right)=\frac{2}{\sqrt{i \tau}},
$$

where the principal branch has been considered for computing $\sqrt{i}$. 
The final result is thus

$$
g(\tau)= \begin{cases}0 & \text { for } \tau<0 \\ \frac{1}{i} \sqrt{\frac{1}{2 \pi i \tau}} \exp \left(i \frac{\kappa_{\| 0}^{2}}{2} \tau\right) . & \text { otherwise }\end{cases}
$$

\section{References}

1. S. Alberti, J.-Ph. Ansermet, K. A. Avramides, F. Braunmueller, P. Cuanillon, J. Dubray, D. Fasel, J.Ph. Hogge, A. Macor, E. de Rijk, M. da Silva, M. Q. Tran, T. M. Tran, and Q. Vuillemin, Experimental study from linear to chaotic regimes on a terahertz-frequency gyrotron oscillator, Phys. of Plasmas, 19, 123102 (2012).

2. S. Alberti, F. Braunmueller, T. M. Tran, J. Genoud, J.-P. Hogge, M. Q. Tran, and J.-P. Ansermet, Nanosecond Pulses in a THz Gyrotron Oscillator Operating in a Mode-Locked Self-Consistent QSwitch Regime, Phys. Rev. Lett. 111, 205101 (2013).

3. S. Alberti, T. Tran, K. Avramides, F. Li, and J. P. Hogge, Gyrotron parasitic-effects studies using the time-dependent self- consistent monomode code TWANG, in Infrared, Millimeter and Terahertz Waves (IRMMW-THz), 2011 36th International Conference on (2011) pp. 12.

4. K. Avramides et al., On the numerical scheme employed in gyrotron interaction simulations, EPJ Web of Conf., 32, 04016 (2012).

5. M. Botton, et al., MAGY: a time-dependent code for simulation of slow and fast microwave sources, IEEE Trans. Plasma Sci., 26(3), 882, 1998.

6. N. S. Ginzburg, G. S Nusinovich, and N. A. Zavolsky, Theory of non-stationary processes in gyrotrons with low Q resonators, International Journal of Electronics, 61(6):881, 1986.

7. C. Wu, K.A Avramidis, M. Thumm, J. Jelonnek, An Improved Broadband Boundary Condition for the RF Field in Gyrotron Interaction Modeling, IEEE Trans. Microw. Theory Techn., 63(8):2459, 2015.

8. K. A. Avramidis, Z.C. Ioannidis, S.Kern, A. Samartsev, I. Gr. Pagonakis, I.G. Tigelis and J. Jelonnek, A comparative study of dynamic after-cavity interaction in gyrotrons, Phys. of Plasmas, 22, 053106 (2015).

9. F. Braunmueller, T. M. Tran, Q. Vuillemin, S. Alberti, J. Genoud, J-Ph. Hogge, and M. Q. Tran, TWANG-PIC, a gyro-averaged 1D particle-in-cell code for self-consistent gyrotron simulations. Phys. of Plasmas, 22, 063115 (2015).

10. S. Alberti, et al., European high-power CW gyrotron development for ECRH systems, Fusion Engineering and Design, 53, (2001), 387-397.

11. F. Braunmueller, T. M. Tran, S. Alberti, J.-P. Hogge, and M. Q. Tran, Moment-based, self-consistent linear analysis of gyrotron oscillators, Phys. of Plasmas, 21, 043105 (2014).

12. Carl de Boor, A practical guide to Splines, Applied Mathematica Science, Springer (1978).

13. M. Abramowitz and I. A. Stegun, Handbook of Mathematical Functions, (Dover Publications, Inc., New York, 1964).

14. M. Thumm et al., EU Megawatt-Class 140GHz CW Gyrotron, IEEE Trans. on Plasma Science, 35(2), (2007), p.143.

15. J. Jelonnek et al., From Series Production of Gyrotrons for W7-X Towards EU-1 MW Gyrotrons for ITER, IEEE Trans. on Plasma Science, 42(5) (2014), p.1135. 